\title{
Liver cytochrome P450 system as affected by endophyte-infected tall fescue seed extracts and ergot alkaloids
}

\author{
Ali S. Moubarak*, Zelpha B. Johnson, Charles F. Rosenkrans Jr. \\ Department of Animal Sciences, University of Arkansas, Fayetteville, USA; Corresponding Author: moubarak@uark.edu
}

Received 25 September 2011; revised 29 November 2011; accepted 20 December 2011

\begin{abstract}
Endophyte infected tall fescue $(E+)$ is the base diet for nearly all beef cattle in the southern USA. It has been linked to a variety of toxicological conditions due to the presence of large numbers of ergot alkaloids. This study was designed to investigate the effects of $\mathrm{E}+$ seed extract and selected ergot alkaloids on the detoxification pathway by cytochrome P450 (CYP3A4) enzyme system. Tests were performed using the P450Glo CYP3A4 enzyme activity kit (Promega, WI), according to the manufacturer's manual. Luminescence was measured using a single tube TD20/20 luminometer. Endophyte infected tall fescue seed was extracted with 50/50 methanoll $25 \mathrm{mM}$ ammonium carbonate, cleaned and concentrated on Strata-X reversed phase column (Phenomenex). The extracts were evaluated on an HPLC, and then tested using a serial dilution method. Commercially available ergonovine (EN), ergocorine (ER), bromocryptine (BC) and ergocryptine (EC) were tested individually using 0 to $44 \mathrm{nM}$ concentrations. Seed extract of $\mathrm{E}+$ produced a significant $(P<0.05)$ dose dependent inhibition of CYP3A4 enzyme activity similar to that produced by the commercially available ergot alkaloids EC, ER, BC and EN which inhibited CYP3A4 enzyme activity in a significant $(P<$ $0.05)$ dose dependent manner with EC being most potent, followed by ER, BC, and then EN (70\%, $40 \%, 30 \%$ and $10 \%$ at $44 \mathrm{nM}$ concentration). The similarity of the inhibition curves of seed extract to that of the commercially available ergot alkaloids suggests a related mode of action and that the use of such ergot alkaloids and CYP3A4 assay is a good model to study the toxicity of tall fescue. Furthermore, it provides the foundation to identify the individual toxic components of purified endophyte infected tall
\end{abstract}

fescue extract.

Keywords: Seed Extract; Tall Fescue; CYP3A4; Ergot Alkaloid

\section{INTRODUCTION}

Tall fescue grass is considered to be the primary diet for nearly all beef cattle in the southern United States and has been linked to the incidence of fescue toxicosis syndrome due to the presence of ergot alkaloids. Cytochrome P450 (CYP) enzyme systems play a key role in the biotransformation of many endogenous and exogenous compounds including both toxins and drugs [1] and [2]. The CYP enzyme family consists of a large number of proteins with different substrate specificities and catalytic properties which are membrane-bound, mostly localized to the endoplasmic reticulum and in mitochondrial inner membranes. Ergot alkaloids found in tall fescue are a large family of chemical compounds with two major subfamilies. These are the tetracyclic ergoline nucleus group, such as lysergic acid ethylamide, ergonovine, and lysergic acid, and the ergopeptide alkaloids group which share the tetracyclic ergoline nucleus in addition to tricyclic amino acids such as ergotamine, ergocryptine, and bromocriptine. The metabolism of ergot alkaloids, such as bromocryptine, dihydroergotamine, and other structurally similar ergot derivatives is mediated mainly by CYP3A4 [3]. Moochhala et al. (1989) [4] reported that bromocryptine interferes with P450-dependent oxidative metabolism of xenobiotics. Later it was demonstrated that cytochrome P450 3A exhibits a particularly high affinity for ergopeptides. Ergot alkaloids have been shown to be metabolized by CYP3A; however, information on the effects of these alkaloids on CYP3A activity is very limited. The metabolism of ergot alkaloids has been linked to CYP3A; therefore, activation or inhibition of the induction process of such enzyme systems can have severe consequences on the metabolism of the ergot alkaloids. Witkamp et al. (1995) [5] reported that tiamu- 
lin, a semi-synthetic antibiotic frequently used in agricultural animals, strongly inhibited the hydroxylation rate of testosterone at the 6 beta-position via the formation of a cytochrome P450 3A4 metabolic intermediate complex in both microsomes and hepatocytes. We have reported previously [6] the lack of induction effects of dihydroergotamine and ergonovine on rat CYP3A. The endophyte-infected seed extract treatment was found to suppressed prolactin secretion and the endophyte-free seed extract treatment had no effect on prolactin secretion by rat anterior pituitaries [7]. This study was designed to examine the effects of seed extract of endophyte infected and non infected tall fescue and selected ergot alkaloids ergnovine (EN), ergocorine (ER), bromocryptine (BC) and ergocryptine (EC) on the detoxification pathway by cytochrome P450 (CYP3A4) enzyme system in vitro.

\section{MATERIALS AND METHODS}

All the chemicals and reagents used in these experiments were of the highest quality available and were purchased from Sigma Chemical Co. (St. Louis, MO) unless stated otherwise. Tests were performed using the P450-Glo CYP3A4 enzyme activity kit (Promega, WI), according to the manufacturer's manual. Luminescence was measured using a single tube TD20/20 illuminometer. Briefly, $12.5 \mu \mathrm{l}$ of test compound (seed extract or pure pushed ergot alkaloids) was added per well of a 96-well plate, followed by $12.5 \mu \mathrm{l}$ of the 4 times concentrated CYP450 reaction mixture with and without CYP450 for the blank reactions. Then the plate was pre-incubated at $37^{\circ} \mathrm{C}$ for 10 minutes. The reaction was initiated by adding $25 \mu \mathrm{l}$ of the 2 times concentrated NADPH regeneration system and after 30 minutes incubation at $37^{\circ} \mathrm{C}, 50 \mu$ of reconstituted luciferin detection reagent was added. The plate was mixed for 10 seconds on an orbital shaker and incubated at room temperature for 20 minutes to stabilize the luminescent signal. Then the luminescence was recorded using Perkin Elmer 1420 Victor 3 V.

Endophyte infected tall fescue seed $(\mathrm{E}+$ ) was extracted with 50/50 methanol/25 mM ammonium carbonate (10 $\mathrm{ml} / \mathrm{gm}$ ) for one hour, cleaned and concentrated on Strata-X reversed phase column (Phenomenex). The extracts were evaluated by a Millennium ${ }^{32}$ Workstation HPLC system with auto-sample injector and a gradient programmer. The detection was accomplished using an Applied Biosystems 980 programmable fluorescence detector (excitation at $250 \mathrm{~nm}$ and emission at $370 \mathrm{~nm}$ long pass filter). Separation was conducted on a $3 \times 3$ CR C18 cartridge column using acetonitrile and $2.6 \mathrm{mM}$ ammonium carbonate in $10 \%$ methanol gradient elution at $1 \mathrm{ml} / \mathrm{min}$ flow rate. The extracts were evaluated using $80 \%$ serial dilution method, and the commercially available EC, ER, $\mathrm{BC}$ and EN were tested individually using 0 to $44 \mathrm{nM}$ concentrations.

\section{RESULTS AND DISCUSSION}

The HPLC chromatogram in Figure 1 shows the presence of number of ergot alkaloids in endophyte infected tall fescue. These compounds range from very hydrophilic to very hydrophobic. The non-infected tall fescue seed extract is lacking any of these ergot alkaloids. The toxins found in seed extract of endophyte infected tall fescue inhibited CYP3A4 enzyme activity (Figure 2) in an action similar to that of commercially available ergot alkaloids (EC, ER, and BC) (Figure 3). Seed extract of $\mathrm{E}+$ produced a (Figure 2) dose dependent inhibition (59 and $81 \%$ inhibition at 11 and $100 \%$ of extract concentrations, respectively $(P<0.05)$ of CYP3A4 enzyme activity. Non-infected tall fescue seed extract $(\mathrm{E}-)$ produced no significant effect on CYP3A4 activity due to the lack of the toxins (Figures 1 and 2).

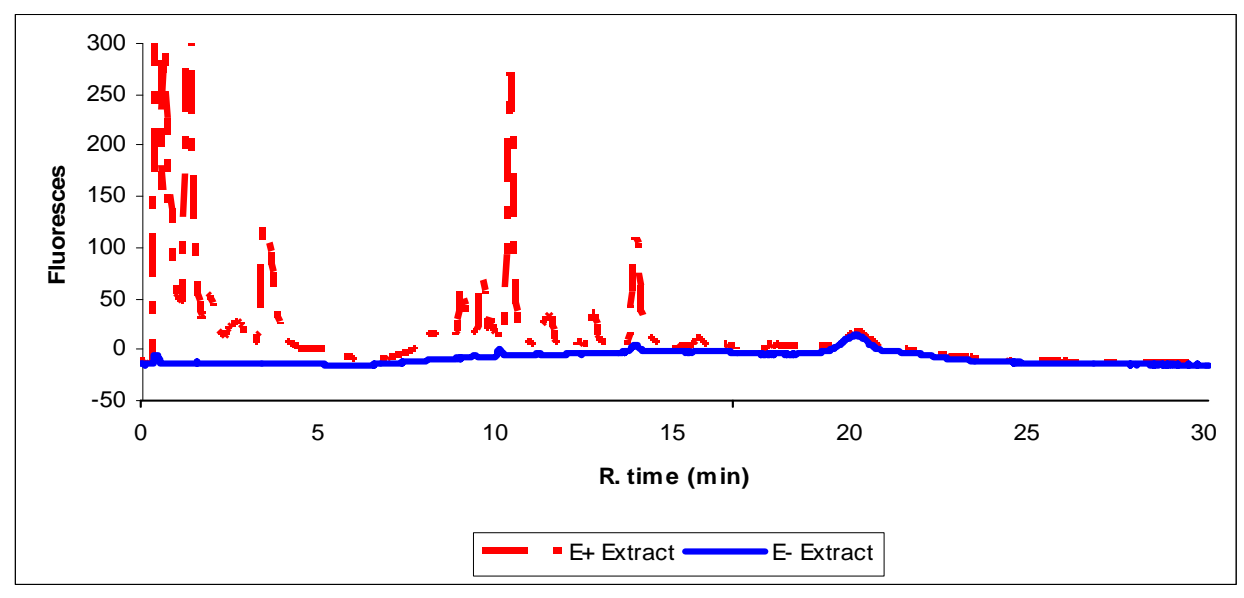

Figure 1. HPLC chromatogram showing the typical profile of seed extract of endophyte infected $(\mathrm{E}+)$ and noninfected $(\mathrm{E}-)$ tall fescue showing some of ergot alkaloids in the $\mathrm{E}+$ and the lack of them in the E-extract. 


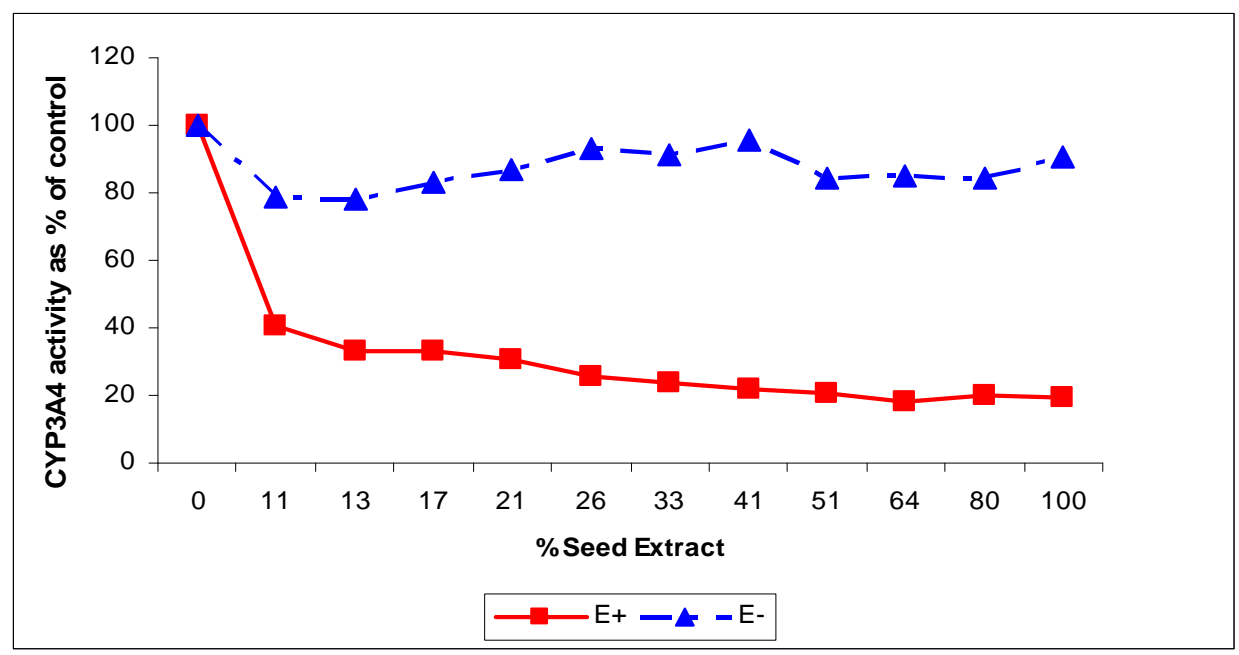

Figure 2. Effects of increasing concentrations of seed extract of endophyte infected $(\mathrm{E}+)$ and noninfected (E-) tall fescue tall fescue on the in vitro activity of CYP3A4. For E+, CYP3A4 activity is reduced at all concentrations of seed extract from the control $0 \%$ seed extract; $P<0.05$ ).

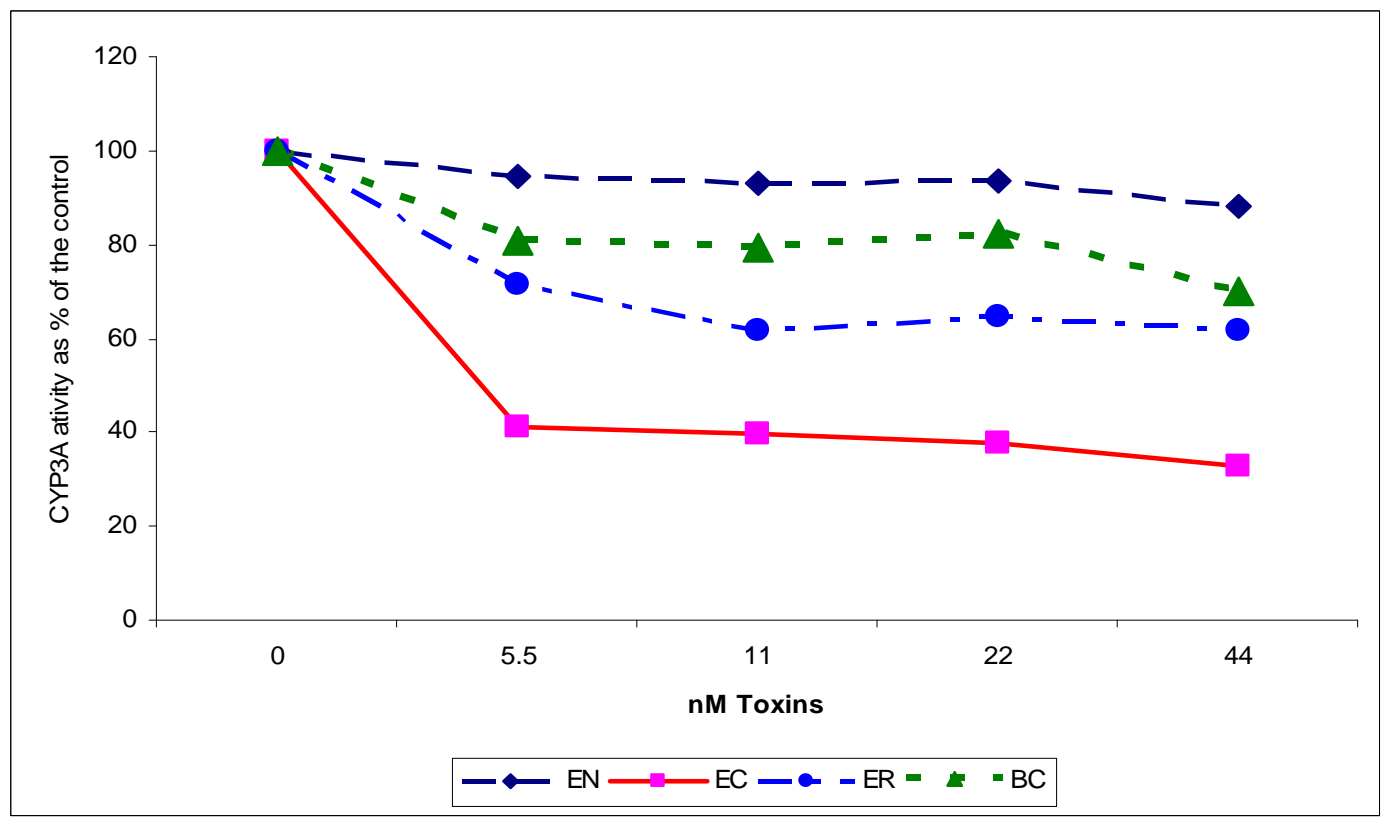

Figure 3. Effects of increasing concentrations of ergonovine (EN), ergocorine (ER), bromocryptine (BC) and ergocryptine (EC) on the in vitro activity of CYP3A4. For ER, BC, and EC, CYP3A activity is reduced from the control ( 0 amount of toxin; $P<0.05$ ).

Ergocryptine, ER, and BC inhibited CYP3A4 enzyme activity in a significant $(P<0.05)$ dose dependent manner with EC being most potent, followed by ER, and then BC $(70 \%, 40 \%$, and $30 \%$ at $44 \mathrm{nM}$ concentration) (Figure 3). The EN effect was not significant. The similarity of the inhibition curves and the chemical structure of toxins found in seed extract to that of the commercially available ergot alkaloids suggest that the toxins found in $\mathrm{E}+$ appear to target the same location on the CYP3A4 and follow the same mechanism of inhibition. The con- nection between the effects of fescue toxins and the effects of the single ergot alkaloids, EC, ER, BC and EN, on CYP enzyme systems has been established. Such association helps in understanding of the effects of $\mathrm{E}+$ in general and lays the foundation to study the detailed effects of each single component of fescue toxins on liver detoxification mechanisms. Also the use of P450-Glo CYP3A4 enzyme activity kit appeared to be a good instrument to study and identify the toxic individual components of purified endophyte infected tall fescue ex- 
tract.

\section{REFERENCES}

[1] Porter, T.D. and Coon, M.J. (1991) Cytochrome P-450. Multiplicity of isoforms, substrates, and catalytic and regulatory mechanisms. Journal of Biological Chemistry, 266, 13469-13472.

[2] Pollock, B.G. (1994) Recent developments in drug metabolism of relevance to psychiatrists. Harvard Review of Psychiatry, 2, 204-213. doi:10.3109/10673229409017138

[3] Ball, S.E., Maurer, G., Zollinger, M., Ladona, M. and Vicker, A.E. (1992) Characterization of the cytochrome $\mathrm{P}-450$ gene family responsible for the N-dealkylation of the ergot alkaloid CQA 206-291 in humans. Drug Metabolism and Disposition, 20, 56-63.

[4] Moochhala, S.M., Lee, E.J., Hu, G.T., Koh, O.S. and Becket, G. (1989) Effects of bromocryptine on hepatic cytochrome P-450 monooxygenase system. Japanese Journal of Pharmacology, 49, 285-291. doi:10.1254/jip.49.285

[5] Witkamp, R.F., Nijmeijer, S.M., Monshouwer, M. and Van Miert, A.S. (1995) The antibiotic tiamulin is a potent inducer and inhibitor of cytochrome P4503A via the formation of a stable metabolic intermediate complex. Studies in primary hepatocyte cultures and liver microsomes of the pig. Drug Metabolism and Disposition, 23, 542547.

[6] Moubarak, A.S., Rosenkrans, C.F. Jr. and Johnson, Z.B. (2003) Modulation of Cytochrome P450 Metabolism by Ergonovine and Dihydroergotamine. Veterinary \& $\mathrm{Hu}$ man Toxicology, 45, 6-9.

[7] Strickland, J.R, Cross, D.L, Jenkins, T.C., Petroski, R.J. and Powell, R.G. (1992) The effect of alkaloids and seed extracts of endophyte-infected tall fescue on prolactin secretion in an in vitro rat pituitary perfusion system. Journal of Animal Science, 70, 2779-2786. 